\section{CardioRenal Medicine}

\title{
Clinical and Operative Determinants of Acute Kidney Injury after Cardiac Surgery
}

\author{
Faeq Husain-Syed a b Maria Giovanna Quattrone ${ }^{c} \quad$ Fiorenza Ferrari $^{b}$, d \\ Pércia Bezerra $^{b}$ Salvador Lopez-Giacoman ${ }^{b}$ Tommaso Hina Danesi ${ }^{\mathrm{e}}$ \\ Sara Samoni ${ }^{b}$ Massimo de Cal ${ }^{b}$ Gökhan Yücel ${ }^{a}{ }^{f} \quad$ Babak Yazdani ${ }^{a}, g$ \\ Werner Seeger $^{a}$ Hans-Dieter Walmrath ${ }^{a}$ Horst-Walter Birk ${ }^{a}$ \\ Loris Salvador $^{\text {h }}$ Claudio Ronco ${ }^{\text {b, }}$ i \\ ${ }^{a}$ Division of Nephrology, Pulmonology and Critical Care Medicine, Department of \\ Internal Medicine II, University Hospital Giessen and Marburg, Giessen, Germany; \\ bInternational Renal Research Institute of Vicenza, Department of Nephrology, Dialysis and \\ Transplantation, San Bortolo Hospital, Vicenza, Italy; ${ }^{C}$ Anesthesiologist and Biostatistician \\ Doctor, Turin, Italy; d Department of Intensive Care, I.R.C.C.S. Policlinico San Matteo, \\ Pavia, Italy; ${ }^{e}$ Department of Cardiac Surgery, San Bortolo Hospital, Vicenza, Italy; ${ }^{f}$ First \\ Department of Medicine, Faculty of Medicine, University Medical Center Mannheim, \\ University of Heidelberg, Mannheim, Germany; ${ }^{9}$ Fifth Department of Medicine, Faculty \\ of Medicine, University Medical Centre Mannheim, University of Heidelberg, Mannheim, \\ Germany; ${ }^{h}$ Department of Clinical Chemistry and Hematology Laboratory, San Bortolo \\ Hospital, Vicenza, Italy; 'Department of Medicine (DIMED), University of Padua, Padua, Italy
}

\section{Keywords}

Cardiopulmonary bypass · Diagnostic performance $\cdot$ Minimally invasive surgery $\cdot$ Renal failure - Thoracic surgery

\begin{abstract}
Introduction: Cardiac surgery-associated acute kidney injury (CSA-AKI) is associated with increased morbidity and mortality. Objectives: We aimed to identify potentially modifiable risk factors for CSA-AKI. Methods: This was a single-center retrospective cohort study of 495 adult patients undergoing cardiac surgery. AKI was diagnosed and staged using full KDIGO criteria incorporating baseline serum creatinine (SC) levels and correction of postoperative SC levels for fluid balance. We examined the association of routinely available clinical and laboratory
\end{abstract}

Trial Registration: www.ClinicalTrials.gov (NCT03102528). Registered retrospectively on March 30, 2017. 
data with AKI using multivariate logistic regression modeling. Results: A total of 103 (20.8\%) patients developed AKI: 16 (15.5\%) patients were diagnosed with AKI upon hospital admission, and 87 (84.5\%) patients were diagnosed with CSA-AKI. Correction of SC levels for fluid balance increased the number of AKI cases to 104 (21.0\%), with 6 patients categorized to different AKI stages. Univariate logistic regression analysis identified five preoperative (age, sex, diabetes mellitus, preoperative systolic pulmonary arterial pressure [PSPAP], acute decompensated heart failure) and five intraoperative predictors of AKI (age, sex, red blood cell [RBC] volume transfused, use of minimally invasive surgery, duration of cardiopulmonary bypass). When all preoperative and intraoperative variables were incorporated into one model, six predictors remained significant (age, sex, use of minimally invasive surgery, RBC volume transfused, PSPAP, duration of cardiopulmonary bypass). Model discrimination performance showed an area under the curve of 0.69 for the model including only preoperative variables, 0.76 for the model including only intraoperative variables, and 0.77 for the model including all preoperative and intraoperative variables. Conclusions: Use of minimally invasive surgery and therapies mitigating PSPAP and intraoperative blood loss may offer protection against CSA-AKI.

(C) 2020 The Author(s)

Published by S. Karger AG, Basel

\section{Introduction}

Cardiac surgery-associated acute kidney injury (CSA-AKI) occurs in approximately onethird of patients and represents one of the most important negative predictors of outcome in this population [1]. CSA-AKI is associated with a risk of short- and long-term mortality [2]. Still, there are few tools to predict CSA-AKI in the early postoperative period.

Recognizing general and center-specific risk factors (especially modifiable risk factors) could help clinicians to mitigate the risk of CSA-AKI and CSA-AKI-associated complications. Novel urinary biomarkers have been favored for early postoperative recognition of CSA-AKI; however, these do not measure risk before exposure and may not be available routinely [3]. Several tools to stratify the risk of AKI have been introduced into clinical practice and have shown good discrimination in most of the populations tested but have been tested only for severe CSA-AKI necessitating dialysis [4]. Thus, the benefits of using these risk models may be limited due to the small number of patients needing dialysis after surgery.

Few research teams have attempted to predict overall CSA-AKI using full Kidney Disease: Improving Global Outcomes (KDIGO) consensus criteria [5]. Two models have been proposed: Jiang et al. [6] included variables from the preoperative to early postoperative period to predict CSA-AKI in a Chinese population; Chen et al. [7] used preoperative variables only to predict AKI after isolated coronary artery bypass grafting in a Taiwanese population. No research team has utilized full KDIGO consensus criteria for AKI to incorporate a reference value for the serum creatinine (SC) level at baseline and correction of the postoperative SC level for fluid balance. Both factors are important because the AKI diagnosis relies on the baseline SC level, which reflects steady-state kidney function most appropriately but is often unavailable upon hospital admission [8]. This is particularly true for patients undergoing emergency or urgent surgical procedures, who often exhibit upstaging of AKI if the reference baseline SC level is taken into account. Also, fluid accumulation seen typically within the first days after cardiac surgery may dilute SC and delay the diagnosis and staging of AKI [9].

We sought to determine the utility of routinely available preoperative and intraoperative clinical and laboratory data for the prediction of CSA-AKI. AKI was diagnosed taking the baseline SC level and correction of the postoperative SC level for fluid balance into account. 
Fig. 1. Study flowchart. Source population included all adult patients undergoing cardiac surgery. CKD, chronic kidney disease; SC, serum creatinine.

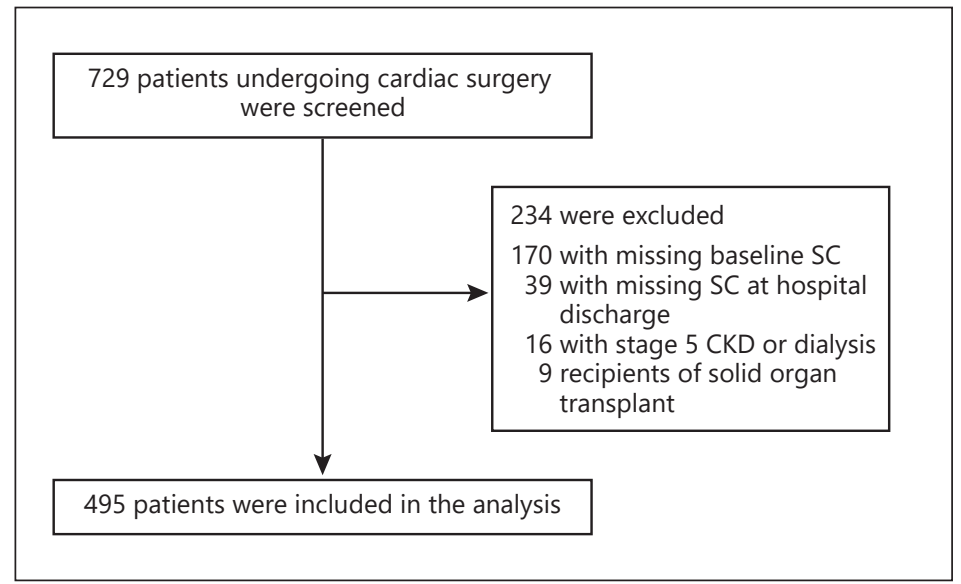

\section{Material and Methods}

\section{Study Design and Participants}

This was a single-center retrospective cohort study of adult patients undergoing cardiac surgery between November 2014 and October 2015 at San Bortolo Hospital. We analyzed data on 729 adult patients undergoing cardiac surgery (elective, urgent, and emergency; coronary artery bypass, valve replacements, combined, or other surgery) (Fig. 1). Approval of the study protocol was obtained from the Human Research Ethics Committee of San Bortolo Hospital (Vicenza, Italy; 79/16 D). The study protocol complied with the tenets of the Declaration of Helsinki.

Cardiac surgery was performed as conventional sternotomic or minimally invasive cardiac surgery. Conventional sternotomic approach was the standard of care for (a) isolated coronary artery bypass graft; (b) coronary artery bypass graft combined with other surgeries (valvular surgery, maze procedure, replacement of the ascending aorta or surgery of atrial septal defects and cardiac tumors); and (c) replacement of the ascending aorta with or without valve surgery. Minimally invasive surgery included both mini-sternotomic and endoscopic surgical approaches. The endoscopic surgical approach was the standard of care for mitral valve and tricuspid valve surgeries, and surgery of atrial septal defects and cardiac tumors with no patient selection and without additional preoperative examinations. $60 \%$ of aortic valve surgeries were performed with endoscopic surgical approach; the other $40 \%$ were performed with a mini-sternotomic approach. Regardless of the surgery, the standard setup for the minimally invasive group included one 3-cm working port (periareolar incision for mitral and tricuspid valve surgery, and atrial septum defect surgery; prepectoral incision for isolated aortic valve or combined aortic and other valve surgery) and two 5-mm miniports for the introduction of the $30^{\circ}$ thoracoscope and the left ventricle vent-line. In all patients undergoing minimally invasive surgery, cardiopulmonary bypass (CPB) was achieved from femoral vessels with retrograde perfusion.

Patients were excluded if (i) the baseline SC level or SC level within 3 days before hospital discharge was not available; (ii) they had stage-5 chronic kidney disease (CKD) [10]; (iii) they were recipients of dialysis before surgery or solid-organ transplantation.

Heart failure was diagnosed according to current guidelines (see also online suppl. material; for all online suppl. material, see www.karger.com/doi/10.1159/000507777) [11]. New York Heart Association (NYHA) functional class was assessed 1 day before cardiac surgery according to current guidelines [11]. Echocardiography was performed by experienced echocardiographers 1 day before cardiac surgery using a Vivid E9 system (GE Healthcare, Wauwatosa, WI, USA). Left ventricular ejection fraction, the ratio of mitral inflow velocity to lateral annular relaxation velocity, and preoperative systolic pulmonary arterial pressure (PSPAP) were measured as recommended [12].

\section{Laboratory Methods}

SC levels were obtained from the Department of Clinical Chemistry and Hematology Laboratory within San Bortolo Hospital to avoid inter-laboratory variations. The SC level was measured by an enzymatic method (IL test ${ }^{\mathrm{TM}}$; Instrumentation ${ }^{\circledR}$ Laboratory, Milan, Italy) on an ILab650 analyzer (Werfen, Barcelona, Spain). The glomerular filtration rate was estimated using the CKD Epidemiology Collaboration equation [13]. 
Table 1. Patient characteristics

\begin{tabular}{|c|c|c|c|c|}
\hline & $\begin{array}{l}\text { Total } \\
(n=495)\end{array}$ & $\begin{array}{l}\text { Non-AKI group } \\
(n=392)\end{array}$ & $\begin{array}{l}\text { AKI group } \\
(n=103)\end{array}$ & $p$ value* \\
\hline \multicolumn{5}{|l|}{ Demographics } \\
\hline Age, years & $64 \pm 13$ & $63 \pm 13$ & $68 \pm 12$ & 0.0001 \\
\hline Male sex & $335(67.7 \%)$ & $273(69.1 \%)$ & $62(60.2 \%)$ & 0.034 \\
\hline Body mass index, $\mathrm{kg} / \mathrm{m}^{2}$ & $24.5 \pm 4.6$ & $24.4 \pm 4.9$ & $24.8 \pm 4.2$ & 0.456 \\
\hline \multicolumn{5}{|l|}{ Comorbidities } \\
\hline Hypertension [yes] & $394(79.6 \%)$ & $306(78.1 \%)$ & $88(85.4 \%)$ & 0.098 \\
\hline Atrial fibrillation [yes] & $95(19.2 \%)$ & $70(17.9 \%)$ & $25(24.3 \%)$ & 0.141 \\
\hline Diabetes mellitus [yes] & $61(12.3 \%)$ & $37(9.4 \%)$ & $24(23.3 \%)$ & $<0.0001$ \\
\hline Heart failure [yes] & $16(12.3 \%)$ & $7(1.8 \%)$ & $9(8.7 \%)$ & $<0.0001$ \\
\hline Coronary heart disease [yes] & $141(28.5 \%)$ & $95(23.2 \%)$ & $46(44.7 \%)$ & $<0.0001$ \\
\hline Peripheral vasculopathy [yes] & $82(16.6 \%)$ & $51(13.0 \%)$ & $31(30.1 \%)$ & $<0.0001$ \\
\hline Dyslipidemia [yes] & $226(45.7 \%)$ & $162(41.3 \%)$ & $64(62.1 \%)$ & $<0.0001$ \\
\hline Previous myocardial infarction [yes] & $54(11.9 \%)$ & $33(8.4 \%)$ & $21(20.4 \%)$ & 0.0012 \\
\hline \multicolumn{5}{|l|}{ Medication } \\
\hline Antiplatelet [yes] & $145(29.3 \%)$ & $105(26.8 \%)$ & $40(87.4 \%)$ & 0.0173 \\
\hline Beta-blocker [yes] & $252(50.9 \%)$ & $190(48.5 \%)$ & $62(60.2 \%)$ & 0.0342 \\
\hline ACEi or ARB [yes] & $268(54.1 \%)$ & $212(54.1 \%)$ & $56(54.4 \%)$ & 0.958 \\
\hline Statin [yes] & $184(37.2 \%)$ & $130(33.2 \%)$ & $54(52.4 \%)$ & $<0.0001$ \\
\hline Diuretic $[y e s]^{\mathrm{a}}$ & $486(98.2 \%)$ & $385(98.2 \%)$ & $101(98.1 \%)$ & 0.140 \\
\hline Aldosterone antagonist [yes] & $42(8.5 \%)$ & $32(8.2 \%)$ & $10(9.7 \%)$ & 0.616 \\
\hline Preoperative intravenous contrast media [yes] ${ }^{\mathrm{b}}$ & $63(13.1 \%)$ & $39(9.9 \%)$ & $24(23.3 \%)$ & $<0.0001$ \\
\hline \multicolumn{5}{|l|}{ Baseline clinical data } \\
\hline Leukocytes, $\times 10^{9} / \mathrm{L}$ & $6.4 \pm 1.9$ & $6.4 \pm 1.9$ & $6.4 \pm 1.8$ & 0.9408 \\
\hline Hemoglobin, g/dL & $13.8 \pm 1.7$ & $14.0 \pm 1.7$ & $13.3 \pm 1.8$ & 0.001 \\
\hline Platelets, $\times 10^{9} / \mathrm{L}$ & $213 \pm 50$ & $211.4 \pm 48.9$ & $217.63 \pm 52.9$ & 0.259 \\
\hline Albumin, g/dL & $4.0 \pm 1.1$ & $4.0 \pm 1.2$ & $3.9 \pm 0.2$ & 0.344 \\
\hline Urea, $\mathrm{mg} / \mathrm{dL}^{\mathrm{c}}$ & $37.6 \pm 10.5$ & $36.7 \pm 9.4$ & $41 \pm 13.3$ & 0.0017 \\
\hline B-type natriuretic peptide, $\mathrm{pg} / \mathrm{mL}$ & $64.5 \pm 32.3$ & $52.1 \pm 27.4$ & $71.3 \pm 38.1$ & $<0.0001$ \\
\hline \multicolumn{5}{|l|}{ NYHA classification } \\
\hline 1 & $134(27.1 \%)$ & $114(29.1 \%)$ & $20(19.4 \%)$ & 0.0024 \\
\hline 2 & $301(60.8 \%)$ & $241(61.5 \%)$ & $60(58.3 \%)$ & \\
\hline 3 & $50(10.1 \%)$ & $32(8.2 \%)$ & $18(17.5 \%)$ & \\
\hline 4 & $10(2.0 \%)$ & $5(1.3 \%)$ & $5(4.9 \%)$ & \\
\hline Left ventricular ejection fraction, $\%$ & $60.5 \pm 9.6$ & $60.83 \pm 9.32$ & $59.19 \pm 10.49$ & 0.1229 \\
\hline $\mathrm{E} / \mathrm{e}^{\prime}$ ratio & $11.7 \pm 4.6$ & $11.0 \pm 2.8$ & $14.3 \pm 4.9$ & 0.045 \\
\hline Systolic pulmonary arterial pressure, $\mathrm{mm} \mathrm{Hg}$ & $33.2 \pm 10.2$ & $33.03 \pm 10.48$ & $33.86 \pm 9.28$ & 0.4656 \\
\hline EuroSCORE II for operative risk, $\%^{\mathrm{d}}$ & $2.86 \pm-4.21$ & $2.26 \pm 2.84$ & $5.16 \pm 6.96$ & $<0.0001$ \\
\hline \multicolumn{5}{|l|}{ Surgical clinical data } \\
\hline Aortic cross-clamp, min & $81.6 \pm 32.1$ & $80.2 \pm 31.19$ & $86.8 \pm 34.9$ & 0.064 \\
\hline Cardiopulmonary bypass time, min & $120.5 \pm 43.3$ & $116.58 \pm 38.7$ & $135.51 \pm 55.3$ & 0.0034 \\
\hline \multicolumn{5}{|l|}{ Procedure } \\
\hline \multicolumn{5}{|l|}{ Conventional surgery [yes] } \\
\hline Coronary artery bypass graft only & $52(10.5 \%)$ & $46(11.7 \%)$ & $6(5.8 \%)$ & 0.384 \\
\hline Combined coronary artery bypass graft and & & & & \\
\hline other surgery ${ }^{\mathrm{e}}$ & $46(9.3 \%)$ & $36(9.2 \%)$ & $10(9.7 \%)$ & 0.67 \\
\hline Replacement of the ascending aorta with & & & & \\
\hline or without valve surgery & $56(11.3 \%)$ & $45(11.5 \%)$ & $11(10.7 \%)$ & 0.344 \\
\hline \multicolumn{5}{|l|}{ Minimally invasive surgery [yes] } \\
\hline Aortic valve surgery & $25(5.0 \%)$ & $10(2.6 \%)$ & $5(4.9 \%)$ & 0.034 \\
\hline Mitral valve surgery & $122(24.7 \%)$ & $88(22.4 \%)$ & $34(33.0 \%)$ & 0.0002 \\
\hline Tricuspid valve surgery & $2(0.4 \%)$ & $1(0.3 \%)$ & $1(1.0 \%)$ & 0.689 \\
\hline Combined valve surgery and other surgery ${ }^{f}$ & $192(38.8 \%)$ & $166(42.3 \%)$ & $36(35.0 \%)$ & 0.234 \\
\hline
\end{tabular}


Table 1 (continued)

\begin{tabular}{|c|c|c|c|c|}
\hline & $\begin{array}{l}\text { Total } \\
(n=495)\end{array}$ & $\begin{array}{l}\text { Non-AKI group } \\
(n=392)\end{array}$ & $\begin{array}{l}\text { AKI group } \\
(n=103)\end{array}$ & $p$ value* \\
\hline Valve reconstruction & $245(49.5 \%)$ & $220(49.5 \%)$ & $25(56.3 \%)$ & 0.157 \\
\hline Valve replacement & $191(38.6 \%)$ & $129(32.9 \%)$ & $62(60.1 \%)$ & $<0.0001$ \\
\hline Bioprosthetic valve & $186(37.6 \%)$ & $126(32.1 \%)$ & $60(58.3 \%)$ & \\
\hline Mechanical valve & $5(1.0 \%)$ & $3(0.8 \%)$ & $2(1.9 \%)$ & \\
\hline Intraoperative diuresis, $\mathrm{mL}$ & $980 \pm 664$ & $995 \pm 821$ & $927 \pm 789$ & 0.359 \\
\hline Surgery fluid balance, $\mathrm{mL}$ & $3,662 \pm 1,295$ & $3,652 \pm 1,199$ & $3,700 \pm 1,303$ & 0.741 \\
\hline Red blood cell volume transfused, mL & $66.1 \pm 194.2$ & $42.9 \pm 157.7$ & $154.4 \pm 278.2$ & $<0.0001$ \\
\hline \multicolumn{5}{|l|}{ ICU clinical data } \\
\hline Mechanical ventilation, days & $1.5 \pm 2.2$ & $1.1 \pm 0.6$ & $2.8 \pm 4.5$ & $<0.0001$ \\
\hline Intra-aortic balloon pump, days & $0.2 \pm 1.0$ & $0.05 \pm 0.4$ & $0.6 \pm 1.9$ & $<0.0001$ \\
\hline Extra-corporeal membrane oxygenation, days & $0.04 \pm 0.5$ & $0 \pm 0$ & $0.2 \pm 1.0$ & $<0.0001$ \\
\hline Pneumonia [yes] & $10(2.0 \%)$ & $3(0.8 \%)$ & $7(6.8 \%)$ & $<0.0001$ \\
\hline Sepsis [yes] & $11(2.2 \%)$ & $3(0.8 \%)$ & $8(7.8 \%)$ & $<0.0001$ \\
\hline Re-intervention [yes] & $23(4.7 \%)$ & $11(2.8 \%)$ & $12(11.7 \%)$ & $<0.0001$ \\
\hline Fluid balance at day $1, \mathrm{~mL}$ & $-599 \pm 847$ & $-709 \pm 823$ & $-177 \pm 810$ & $<0.0001$ \\
\hline Fluid balance day $2, \mathrm{~mL}$ & $-230 \pm 859$ & $-346 \pm 815$ & $218 \pm 882$ & $<0.0001$ \\
\hline Cumulative fluid balance in the ICU, mL & $-1,618 \pm 1,912$ & $-1,679 \pm 1,662$ & $-1,389 \pm 2,657$ & 0.066 \\
\hline Weight difference, $\mathrm{kg}^{\mathrm{g}}$ & $-0.9 \pm 5.4$ & $-1.3 \pm 4.9$ & $0.6 \pm 7$ & 0.002 \\
\hline Furosemide dose per day, mg & $32.5 \pm 58.7$ & $19.7 \pm 16$ & $81.4 \pm 112.7$ & $<0.0001$ \\
\hline Cumulative colloids, mL & $159.4 \pm 293.1$ & $134.4 \pm 245.8$ & $254.4 \pm 416.1$ & 0.018 \\
\hline Transfusion of red blood cells, mL & $251.3 \pm 769.4$ & $116.2 \pm 320$ & $764 \pm 1,461$ & $<0.0001$ \\
\hline ICU stay, h & $78.7 \pm 74.2$ & $65.3 \pm 37.2$ & $129.8 \pm 134.4$ & $<0.0001$ \\
\hline Hospital stay, days & $8.2 \pm 7.0$ & $7.3 \pm 4.9$ & $11.6 \pm 11.5$ & $<0.0001$ \\
\hline In-hospital mortality & $4(0.8 \%)$ & $0(0 \%)$ & $4(3.9 \%)$ & $<0.0001$ \\
\hline
\end{tabular}

ACEi, angiotensin-converting enzyme inhibitor; ARB, angiotensin II receptor blocker; E/e' ratio, ratio of mitral inflow velocity to lateral annular relaxation velocity; ICU, intensive care unit; NYHA, New York Heart Association. Summaries of quantitative variables are the mean \pm standard deviation. For categorical variables, the absolute and relative frequencies (as $\%$, in parentheses) for the categories are presented. For dichotomous variables, the respective category is presented in square brackets. Additional data are provided in online supplementary Table S1. * After application of the Bonferroni correction, $p<$ 0.0009 was considered significant. ${ }^{\text {a }}$ Diuretics include loop diuretics and thiazides. ${ }^{\mathrm{b}}$ Intravenous contrast media within 7 days before surgery. ${ }^{\mathrm{c}}$ To convert the value for urea to blood urea nitrogen, multiply by $0.467 .{ }^{\mathrm{d}}$ The European System for Cardiac Operative Risk Evaluation (EuroSCORE) score is calculated by means of a logistic-regression equation and ranges from 0 to $100 \%$, with higher scores indicating greater risk. ${ }^{e}$ Other surgery includes valvular surgery, maze procedure, replacement of the ascending aorta, surgery of atrial septal defects, and surgery of cardiac tumors. ${ }^{\mathrm{f}}$ Other surgery includes maze procedure, surgery of atrial septal defects, and surgery of cardiac tumors. gICU discharge - hospital admission.

\section{Data Collection}

We abstracted clinical variables from the medical records of patients. Use of renal replacement therapy (RRT) was undertaken according to the discretion of the treating physician. Complications were recorded, and postoperative myocardial infarction and stroke were defined as described previously [14]. The SC level at baseline was considered to be the value within 90 days before surgery, which reflects steady-state kidney function most appropriately [5]. Positive fluid balance and hemodilution were considered in the diagnosis and staging of AKI using the following formula [9]:

Adjusted SC level $=$ SC $\times$ correction factor,

where the correction factor $=($ weight $[\mathrm{kg}]$ upon hospital admission $\times 0.6+\Sigma$ [daily cumulative fluid balance [L]])/hospital admission weight $\times 0.6$.

Use of potentially nephrotoxic drugs (nonsteroidal anti-inflammatory drugs, aminoglycosides, vancomycin, intravenous contrast media) during the study period was recorded. 
End Points

The primary end point was to determine the predictive variables of AKI using multivariate logistic regression models. AKI development was diagnosed within 7 days after cardiac surgery as determined by a Clinical Adjudication Committee using full consensus criteria from KDIGO [5]. In case patients met both SC and urine output criteria, AKI staging was performed according to the highest severity criterion. The secondary end point was the prevalence of renal recovery upon hospital discharge. "Renal recovery" was defined as the absence of any stage of AKI according to the SC level or urine output upon hospital discharge, as described previously [15]. We considered death before hospital discharge to be "non-recovery" because renal recovery without survival is rare and not patient-centered, as suggested by international consensus recommendations [16].

\section{Statistical Analyses}

Descriptive statistics were expressed as the mean \pm standard deviation or median (interquartile range [IQR]) for continuous variables and analyzed by an independent Student's $t$ test. The Shapiro-Wilk test was used to test the Gaussian distribution of the continuous variables. Categorical variables were reported as absolute frequencies and percentages and analyzed by the $\chi^{2}$ test or Fisher's exact test, as appropriate. The Student's $t$ test or $\chi^{2}$ test (or equivalent nonparametric test) was used to compare the two groups: AKI and non-AKI. Successively, we conducted univariate logistic regression for each variable. Variables with $p<0.05$ were considered to be significant. Then, statistically significant or clinically relevant variables (age and sex) were gathered into three groups and used in three stepwise backward multivariate logistic regression analyses: preoperative, intraoperative, and intensive care unit (ICU) stay. The selected models were finally combined in a unitary logistic regression model, which after postestimation analysis included only preoperative and intraoperative variables. All the models were verified with respect to collinearity and the interactions between variables to guard against confounding. The goodness of fit was calculated by the HosmerLemeshow test. The accuracy of the classification was assessed by analyses of receiver operating characteristic (ROC) $\beta^{\prime}$ curves and the Akaike information criterion. The Bonferroni correction was applied to adjust for multiple testing. Analyses were conducted using STATA 14 (Stata Corporation, College Station, TX, USA).

\section{Results}

A total of 495 patients were enrolled in our study (Fig. 1). Patient characteristics at baseline as well as clinical data on the surgical procedure and ICU stay are shown in Table 1 and online supplementary Table S1. None of the patients were perioperatively switched from minimally invasive to conventional surgery. The majority $(82.1 \%)$ of patients with NYHA functional class 1 underwent minimally invasive surgery, with mitral valve surgery, correction of atrial septal defects, and surgery of cardiac tumors as the predominant types of surgery. Overall, 103 (20.8\%) had an episode of AKI during hospitalization: 76 (15.4\%) had stage-1, $18(3.6 \%)$ had stage-2, and $9(1.8 \%)$ had stage-3 AKI (Table 2). All patients with stage-3 AKI required RRT and were diagnosed according to both SC and urine output criteria. Of the 103 patients who experienced AKI, 16 (15.5\%) patients were diagnosed with AKI upon hospital admission, all had emergency or urgent cardiac surgery, whereas the remaining 87 (84.5\%) patients were diagnosed with CSA-AKI within $48 \mathrm{~h}$ after surgery. In addition, $73.8 \%$ of patients were diagnosed and staged according to positive SC criteria for AKI.

By correcting the SC level for fluid balance, the total number of AKI cases increased to 104 patients (21.0\%) (online suppl. Table S2). Two patients who previously had stage-1 AKI were now classified as not having developed AKI; 3 patients with previously did not have AKI were now staged as having stage- $1 \mathrm{AKI}$; and 1 patient who previously had stage- 2 AKI was now classified as having stage-3 AKI.

The mean estimated glomerular filtration rate upon hospital admission for patients who were found to have AKI subsequently was $73.2 \pm 20.2 \mathrm{~mL} / \mathrm{min} / 1.73 \mathrm{~m}^{2}$, and this value was significantly lower than that for people who did not have AKI $(86.95 \pm 16.71 \mathrm{~mL} / \mathrm{min} / 1.73$ 
Table 2. Outcomes

\begin{tabular}{|c|c|c|c|}
\hline & $\begin{array}{l}\text { Non-AKI group } \\
(n=392)\end{array}$ & $\begin{array}{l}\text { AKI group } \\
(n=103)\end{array}$ & $p$ value* \\
\hline \multicolumn{4}{|l|}{ Serum creatinine, $m g / d L^{\mathrm{a}}$} \\
\hline Hospital admission & $0.91 \pm 0.60$ & $0.99 \pm 0.62$ & 0.0005 \\
\hline Postoperative day 1 & $0.91 \pm 0.85$ & $0.83 \pm 0.25$ & 0.9839 \\
\hline Postoperative day 2 & $0.95 \pm 0.55$ & $0.99 \pm 0.35$ & 0.0152 \\
\hline Hospital discharge & $0.82 \pm 0.22$ & $1.06 \pm 0.50$ & $<0.0001$ \\
\hline \multicolumn{4}{|l|}{ Estimated GFR, $\mathrm{mL} / \mathrm{min} / 1.73 \mathrm{~m}^{2 \mathrm{~b}}$} \\
\hline Hospital admission & $86.95 \pm 16.71$ & $73.17 \pm 20.21$ & $<0.0001$ \\
\hline Hospital discharge & $90.6 \pm 16.46$ & $71.18 \pm 24.50$ & $<0.0001$ \\
\hline AKI at hospital admission & - & $16(15.5 \%)$ & $\mathrm{N} / \mathrm{A}$ \\
\hline AKI within $48 \mathrm{~h}$ after surgery & - & $87(84.5 \%)$ & $\mathrm{N} / \mathrm{A}$ \\
\hline AKI stage & & & $\mathrm{N} / \mathrm{A}$ \\
\hline 1 & - & $76(73.8 \%)$ & \\
\hline 2 & - & $18(17.5 \%)$ & \\
\hline 3 & - & $9(8.7 \%)$ & \\
\hline Postoperative renal replacement therapy [yes] & - & $9(8.7 \%)$ & $\mathrm{N} / \mathrm{A}$ \\
\hline Positive AKI criterion & & & $\mathrm{N} / \mathrm{A}$ \\
\hline Serum creatinine & - & $76(73.8 \%)$ & \\
\hline Urine output & - & $14(13.6 \%)$ & \\
\hline Serum creatinine and urine output & - & $13(12.6 \%)$ & \\
\hline Renal recovery state at upon hospital discharge & & & $\mathrm{N} / \mathrm{A}$ \\
\hline Recovery & - & $86(83.5 \%)$ & \\
\hline Non-recovery & - & $17(16.5 \%)$ & \\
\hline
\end{tabular}

AKI, acute kidney injury; GFR, glomerular filtration rate; N/A, not applicable/not available. Summaries of quantitative variables are the mean \pm standard deviation. For categorical variables, the absolute and relative frequencies (as \%, in parentheses) are presented for the categories. For dichotomous variables, the respective category is presented in square brackets. ${ }^{\text {a }}$ To convert the values for serum creatinine to $\mu \mathrm{mol} / \mathrm{L}$, multiply by 88.4. ${ }^{\mathrm{b}}$ The estimated GFR was calculated using the Chronic Kidney Disease-Epidemiology. Collaboration equation [13]. ${ }^{\mathrm{c}} \mathrm{AKI}$ reversal was defined as the absence of any stage of AKI based on the serum creatinine or urine output [15].

$\left.\mathrm{m}^{2} ; p<0.0001\right)$. There was no detectable variability in the proportion of patients who developed AKI among the different types of surgical procedure. However, patients with AKI were older ( $68 \pm 12$ vs. $63 \pm 13$ years; $p<0.001$ ) and more likely to have associated comorbidity (e.g., heart failure, coronary heart disease, peripheral vasculopathy, diabetes mellitus, chronic obstructive pulmonary disease). Overall, patients with AKI were more likely to develop postoperative complications and experience surgical reinterventions. Also, patients with AKI were more likely to require mechanical ventilation, intra-aortic balloon pump and extracorporeal membrane oxygenation for a longer duration in the postoperative period. Furthermore, patients in the AKI group had an increased length of stay in the ICU (129.8 \pm 134.4 vs. $65.3 \pm 37.2 \mathrm{~h} ; p<0.0001)$ and hospital $(11.6 \pm 11.5$ vs. $7.3 \pm 4.9$ days; $p<0.0001)$.

Seventeen $(16.5 \%)$ patients in the AKI group did not recover their renal function at the time of hospital discharge, whereas 3 (33.3\%) cases remained RRT-dependent. Four patients died; all were in the AKI group.

All variables were included in a univariate analysis to predict CSA-AKI. Multivariate logistic analysis was conducted at three time periods: preoperative, intraoperative, and during ICU stay, then finally combined in a conclusive model to predict CSA-AKI (Table 3). 
Table 3. Preoperative predictors, intraoperative predictors, and combined pre- and intraoperative predictors of cardiac surgery-associated acute kidney injury identified by univariate and multivariate logistic regression models

\begin{tabular}{|c|c|c|c|c|}
\hline \multirow[t]{2}{*}{ Predictors } & \multicolumn{2}{|l|}{ Univariate } & \multicolumn{2}{|l|}{ Multiple } \\
\hline & OR $(95 \% \mathrm{CI})$ & $p$ value & OR $(95 \% \mathrm{CI})$ & $p$ value \\
\hline \multicolumn{5}{|l|}{ Preoperative predictors ${ }^{\mathrm{a}}$} \\
\hline Age & $1.51(1.24-1.87)$ & $<0.0001$ & $1.36(1.10-1.68)$ & 0.005 \\
\hline Male sex & $0.66(0.42-1.03)$ & 0.069 & $0.63(0.39-1.02)$ & 0.060 \\
\hline Peripheral vasculopathy & $2.88(1.72-4.81)$ & $<0.0001$ & & \\
\hline Coronary heart disease & $2.52(1.61-3.97)$ & $<0.0001$ & & \\
\hline Diabetes mellitus & $2.92(1.65-5.15)$ & $<0.0001$ & $2.68(1.47-4.90)$ & 0.001 \\
\hline Dyslipidemia & $2.33(1.49-3.64)$ & $<0.0001$ & & \\
\hline Acute decompensated heart failure & $5.27(1.91-14.50)$ & 0.001 & $4.69(1.60-13.74)$ & 0.005 \\
\hline NYHA classification & $1.79(1.29-2.48)$ & $<0.0001$ & & \\
\hline Statin & $2.22(1.43-3.45)$ & $<0.001$ & & \\
\hline Urea & $1.89(1.21-2.96)$ & 0.005 & & \\
\hline Preoperative intra-aortic balloon pump & $7.88(1.42-43.63)$ & 0.018 & & \\
\hline Preoperative intravenous contrast media & $1.58(1.14-2.18)$ & 0.006 & & \\
\hline Preoperative systolic pulmonary arterial pressure & $3.62(1.28-10.27)$ & 0.016 & $3.32(1.15-9.58)$ & 0.026 \\
\hline Carotid stenosis & $2.43(1.18-5.01)$ & 0.016 & & \\
\hline Hemoglobin & $0.78(0.64-0.96)$ & 0.016 & & \\
\hline Antiplatelet & $1.74(1.10-2.74)$ & 0.018 & & \\
\hline Beta-blocker & $1.61(1.03-2.50)$ & 0.035 & & \\
\hline Chronic obstructive pulmonary disease & $2.12(1.01-4.41)$ & 0.046 & & \\
\hline \multicolumn{5}{|l|}{ Intraoperative predictors ${ }^{\mathrm{b}}$} \\
\hline Male sex & $0.66(0.42-1.03)$ & 0.060 & $0.75(0.46-1.25)$ & 0.271 \\
\hline Age & $1.52(1.24-1.87)$ & $<0.0001$ & $1.24(0.99-1.55)$ & 0.058 \\
\hline Urgency of surgery & $7.03(3.36-14.69)$ & $<0.0001$ & & \\
\hline $\mathrm{HCO}_{3}{ }^{-}$level at end of surgery & $1.83(1.18-2.83)$ & 0.007 & & \\
\hline Red blood cell volume transfused & $4.41(2.52-7.73)$ & $<0.0001$ & $2.28(1.23-423)$ & 0.009 \\
\hline Minimally invasive surgery & $0.21(0.13-0.33)$ & $<0.0001$ & $0.26(0.15-0.43)$ & $<0.0001$ \\
\hline Duration of cardiopulmonary bypass & $1.31(1.07-1.59)$ & 0.008 & $1.24(0.01-1.54)$ & 0.045 \\
\hline Intraoperative colloids & $2.01(1.12-3.60)$ & 0.019 & & \\
\hline \multicolumn{5}{|l|}{ Combined pre- and intraoperative predictors ${ }^{c}$} \\
\hline Age & $1.24(0.99-1.55)$ & 0.058 & & \\
\hline Male sex & $0.75(0.46-1.25)$ & 0.241 & & \\
\hline Minimally invasive surgery & $0.26(0.15-0.43)$ & $<0.0001$ & & \\
\hline Preoperative systolic pulmonary arterial pressure & $3.17(1.08-9.29)$ & 0.036 & & \\
\hline Red blood cell volume transfused & $2.28(1.23-4.23)$ & 0.009 & & \\
\hline Duration of cardiopulmonary bypass & $1.24(1.01-1.54)$ & 0.045 & & \\
\hline
\end{tabular}

AUC, area under the curve; NYHA, New York Heart Association; OR, odds ratio; $\mathrm{HCO}_{3}{ }^{-}$, bicarbonate. The table includes only variables that remained significant after multivariate logistic regression analysis. All available variables were included in the univariate analyses, and variables that were significant in the univariate analyses are provided in online supplementary Tables S3-S4.

${ }^{a}$ Analyses of receiver operating characteristic curve showed that preoperative variables predicted AKI with an AUC of 0.688 (Akaike information criterion $=475.27$; goodness of fit $p=0.707$; correctly classified $80.81 \%$; sensitivity $15.53 \%$; specificity $97.96 \%$; the receiver operating characteristic curve is provided in online suppl. Fig. S1).

${ }^{\mathrm{b}}$ Analysis of receiver operating characteristic curve showed that intraoperative variables predicted AKI with an AUC of 0.7575 (Akaike information criterion $=446.08$; goodness of fit $p=0.038$; correctly classified $80.40 \%$; sensitivity $21.36 \%$; specificity $95.92 \%$; the receiver operating characteristic curve is provided in online suppl. Fig. S1).

${ }^{c}$ Analysis of receiver operating characteristic curve showed that combined preoperative and intraoperative variables predicted AKI with an AUC of 0.7652 (Akaike information criterion $=445.69$; goodness of fit $p=0.0305$; correctly classified $80.81 \%$; sensitivity 22.33\%; specificity $96.17 \%$; the receiver operating characteristic curve is provided in online suppl. Fig. S1).

Significant preoperative variables were age, male sex, diabetes mellitus, diagnosis of acute decompensated heart failure, and estimated PSPAP. Significant intraoperative variables were age, male sex, transfusion volume of red blood cells (RBCs), use of minimally invasive surgery, and duration of CPB. Finally, when all preoperative and intraoperative variables were included 


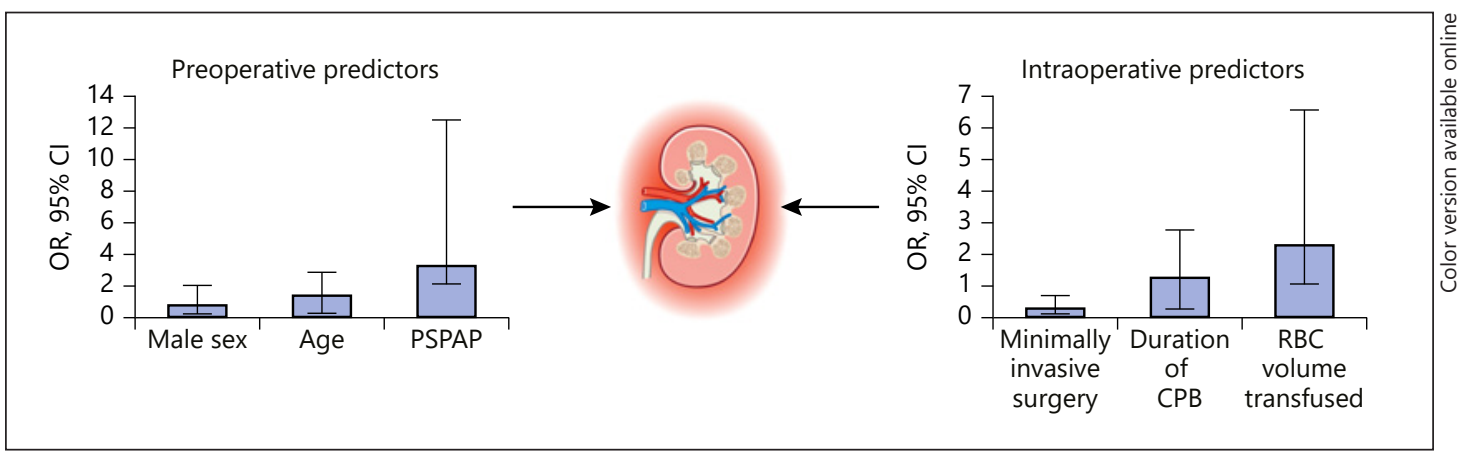

Fig. 2. Clinical and operative determinants of acute kidney injury after cardiac surgery. CPB, cardiopulmonary bypass; OR, odds ratio; PSPAP, preoperative systolic pulmonary arterial pressure; RBC, red blood cell.

in a model, six predictors of AKI remained significant: age, male sex, use of minimally invasive surgery, PSPAP, RBC volume transfused, and duration of CPB (Fig. 2).

Analyses of ROC curves for prediction of CSA-AKI are reported in Table 3 and online supplementary Figure S1. Overall, the three risk models had a moderate-to-good discriminatory power for CSA-AKI, with a C-statistic of 0.69 for the model including only preoperative variables, 0.76 for the model including only intraoperative variables, and 0.77 for the model including preoperative and intraoperative variables. All three models exhibited a good negative predictive value but poor positive predictive value.

Multivariate logistic analysis did not reveal significant predictors of renal recovery upon hospital discharge.

\section{Discussion}

\section{Key Findings}

In this retrospective single-center study, we evaluated the preoperative, intraoperative, and ICU risk factors associated with CSA-AKI development. Overall, 20.8\% of patients undergoing cardiac surgery were diagnosed with AKI. Of these, 15.5\% were diagnosed upon hospital admission when the SC level at baseline was incorporated - all these patients were admitted for emergency or urgent cardiac surgery. Correction of the SC level for fluid balance increased the total number of AKI cases only marginally and changed AKI staging in only 6 patients. In addition, we identified various factors associated with CSA-AKI development, whereas use of minimally invasive surgery, preoperative PASP, and volume of RBC transfusion were identified as potentially modifiable risk factors. Analyses of ROC curves had moderate-to-good discriminatory power for CSA-AKI, with a C-statistic ranging from 0.69 (preoperative variable) to 0.77 (preoperative and intraoperative variables). Finally, none of the collected variables were associated with renal recovery upon hospital discharge.

\section{Relationship with Previous Studies}

The prevalence of AKI in the total cohort and according to stage was similar to that in a recently published meta-analysis involving $>320,000$ patients undergoing cardiac surgery [17]. Interestingly, correction of the SC level for fluid balance did not impact the diagnosis or staging of AKI significantly. This finding is in contrast to the work of Moore et al. [18], which showed an increase in AKI occurrence of $\sim 12 \%$ when adjustment of fluid balance was made, and may reflect inter-hospital differences in intraoperative and postoperative standards of 
care of adult patients undergoing cardiac surgery. Although we detected significant differences in the total amount net fluid balance on postoperative day (POD)1 and POD2 between the AKI group and non-AKI group, these differences were markedly lower compared with those detailed by Moore et al. [18], and may explain the differences in AKI occurrence after adjustment of fluid balance.

As expected, we identified various preoperative and intraoperative variables associated with CSA-AKI development. Similar to a recent study [19], we identified PSPAP as a risk factor for CSA-AKI. Estimated PSPAP is commonly used for the initial evaluation of pulmonary hypertension in clinical practice, but the sensitivity and specificity of echocardiography to accurately estimate pulmonary arterial pressures are modest [20]. Nevertheless, pulmonary hypertension and right-heart failure have emerged as important risk factors for CSA-AKI, with venous congestion as the common cause [21]. Hence, future mechanistic studies, possibly including preoperative right heart catheterization, may offer additional insights whether therapies mitigating PSPAP may offer protection against CSA-AKI.

Also, we identified the use of minimally invasive surgery, which was the predominant surgical technique in our study population, to be associated with a low prevalence of AKI; this is likely because of the low risk of postoperative complications and blood transfusion requirement, lower risk of postoperative systemic immune inflammatory syndrome, accelerated recovery, and low use of potential nephrotoxic medications (e.g., nonsteroidal antiinflammatory drugs) [22]. Reports have indicated the noninferiority of this approach to conventional surgery [23], and recent guidelines recommend minimally invasive surgical procedures where expertise is available with a IIa class of recommendation [24]. Murzi et al. [25] showed a $1 \%$ incidence of CSA-AKI after mitral valve surgery using video-assisted minithoracotomy, although in general CPB and cross-clamping, times are longer in the port-access procedures [26]. The present study confirms the reported renoprotective benefits of minimally invasive surgery, and this approach may be considered as an alternative to standard median sternotomy valve surgery in patients at high risk for CSA-AKI.

In accordance with the literature [27, 28], we identified perioperative transfusion of packed RBCs to be associated with a high OR of developing CSA-AKI. Changes in intraoperative levels of hemoglobin can cause a proportional decrease in the oxygen-carrying capacity of the blood, which leads to impaired oxygen delivery and hypoxia in renal tissue, particularly if anemia is acute or severe [28]. RBC transfusion, however, has also been associated with increased morbidity and mortality, presumably due to infectious and noninfectious risks [27]. Therefore, it is plausible that, by decreasing the incidence of blood transfusions and bleeding complications (e.g., with minimally invasive valve surgery), CSA-AKI and its associated complications may be reduced. Of note, both variables transfusion of packed RBCs and minimally invasive surgery were not collinear in our analysis, emphasizing their independent role in the development of CSA-AKI.

The ability of the model to predict CSA-AKI was worse when compared with previously published and externally validated risk prediction scores, with the C-statistic ranging from 0.81 to $0.85[29,30]$. Unexpectedly, we did not detect variables that influenced renal recovery.

\section{Study Implications}

In line with other studies, our study implied that the pathophysiology of CSA-AKI is complex, and current risk prediction tools show only moderate-to-good calibration, suggesting significant heterogeneity in the underlying populations. However, among potentially modifiable risk factors, use of minimally invasive surgery and therapies mitigating preoperative pulmonary arterial pressure and intraoperative blood loss may offer protection against CSA-AKI. 


\section{Strengths and Limitations of Our Study}

No other research teams have utilized full KDIGO consensus criteria for AKI to incorporate the SC level at baseline and correction of the SC level after surgery for fluid balance for the diagnosis and staging of AKI. The other strength of our study was the assessment of multiple variables at baseline, intraoperatively, and in the ICU. In addition, only few studies evaluated the impact of minimally invasive surgery on CSA-AKI.

Study limitations were its retrospective design, relatively small number of patients, and its single-center location. Thus, our results cannot be extrapolated directly to other patient populations. Also, due to the retrospective nature of the study, complete elimination of selection bias is unlikely. Multivariate logistic regression analyses verified with respect to collinearity and the interactions between variables were performed to minimize the differences in measured confounders; however, unmeasured confounders cannot be accounted for. Preoperative right heart catheterization was not performed to confirm the obtained estimated PSPAP values. In addition, we could not evaluate the association of invasive cardiopulmonary hemodynamics with CSA-AKI.

\section{Conclusions}

The pathophysiology of CSA-AKI appears to be complex and associated with various risk factors, suggesting significant heterogeneity in the underlying population. However, among potentially modifiable risk factors, use of minimally invasive surgery and therapies mitigating PSPAP and intraoperative blood loss may offer protection against CSA-AKI.

\section{Statement of Ethics}

The study was approved by the Human Research Ethics Committee of San Bortolo Hospital (Vicenza, Italy; 79/16 D). The Ethics Committee waived the need for informed consent, as this was a retrospective study.

\section{Disclosure Statement}

None of the authors declare any competing interests.

\section{Funding Sources}

There was no external funding source for the conduct of the study.

\section{Author Contributions}

Study concept and design: F.H.-S., F.F., P.B., S.L.-G., T.H.D., S.S., M.d.C., L.S., C.R. C.R. conceived the concept underlying the study and is the senior author of the study. Literature research and clinical advice: F.F., G.Y., B.Y., H.-W.B., W.S., H.-D.W., L.S. Acquisition, analysis, or interpretation of data: F.H.-S., M.G.Q., F.F., P.B., S.L.-G., T.H.D., S.S., M.d.C., L.S., G.Y., B.Y., H.-W.B., W.S, H.-D.W., L.S., C.R. Adjudication of renal function: S.-L.G., S.S., H.-W.B. Drafting of the manuscript: F.H.-S., M.G.Q., F.F., C.R. Critical revision of the manuscript for important intellectual content: F.H.-S., M.G.Q., F.F., C.R. Statistical analyses: M.G.Q., F.F. Study supervision: C.R. All authors read and approved the final manuscript.

\section{Availability of Supporting Data}

The data sets used and/or analyzed during the current study are available from the corresponding author on reasonable request. 


\section{References}

1 Rydén L, Sartipy U, Evans M, Holzmann MJ. Acute kidney injury after coronary artery bypass grafting and longterm risk of end-stage renal disease. Circulation. 2014 Dec;130(23):2005-11.

2 Wang Y, Bellomo R. Cardiac surgery-associated acute kidney injury: risk factors, pathophysiology and treatment. Nat Rev Nephrol. 2017 Nov;13(11):697-711.

3 Kashani K, Al-Khafaji A, Ardiles T, Artigas A, Bagshaw SM, Bell M, et al. Discovery and validation of cell cycle arrest biomarkers in human acute kidney injury. Crit Care. 2013 Feb;17(1):R25.

4 Huen SC, Parikh CR. Predicting acute kidney injury after cardiac surgery: a systematic review. Ann Thorac Surg. 2012 Jan;93(1):337-47.

5 Kidney Disease: Improving Global Outcomes (KDIGO) Acute Kidney Injury Work Group. KDIGO Clinical Practice Guideline for Acute Kidney Injury. Kidney Int Suppl. 2012;(2):1-138.

6 Jiang W, Teng J, Xu J, Shen B, Wang Y, Fang Y, et al. Dynamic Predictive Scores for Cardiac Surgery-Associated Acute Kidney Injury. J Am Heart Assoc. 2016 Aug;5(8):e003754.

7 Chen SW, Chang CH, Fan PC, Chen YC, Chu PH, Chen TH, et al. Comparison of contemporary preoperative risk models at predicting acute kidney injury after isolated coronary artery bypass grafting: a retrospective cohort study. BMJ Open. 2016 Jun;6(6):e010176.

8 Thomas ME, Blaine C, Dawnay A, Devonald MA, Ftouh S, Laing C, et al. The definition of acute kidney injury and its use in practice. Kidney Int. 2015 Jan;87(1):62-73.

9 Macedo E, Bouchard J, Soroko SH, Chertow GM, Himmelfarb J, Ikizler TA, et al.; Program to Improve Care in Acute Renal Disease Study. Fluid accumulation, recognition and staging of acute kidney injury in critically-ill patients. Crit Care. 2010;14(3):R82.

10 Kidney Disease: Improving Global Outcomes (KDIGO) CKD Work Group. KDIGO 2012 Clinical Practice Guideline for the Evaluation and Management of Chronic Kidney Disease. Kidney Int Suppl. 2013;(3):1-150.

11 Ponikowski P, Voors AA, Anker SD, Bueno H, Cleland JG, Coats AJ, et al.; ESC Scientific Document Group. 2016 ESC Guidelines for the diagnosis and treatment of acute and chronic heart failure: the Task Force for the diagnosis and treatment of acute and chronic heart failure of the European Society of Cardiology (ESC)Developed with the special contribution of the Heart Failure Association (HFA) of the ESC. Eur Heart J. 2016 Jul;37(27): 2129-200.

12 Rudski LG, Lai WW, Afilalo J, Hua L, Handschumacher MD, Chandrasekaran K, et al. Guidelines for the echocardiographic assessment of the right heart in adults: a report from the American Society of Echocardiography endorsed by the European Association of Echocardiography, a registered branch of the European Society of Cardiology, and the Canadian Society of Echocardiography. J Am Soc Echocardiogr. 2010 Jul;23(7):685-713.

13 Levey AS, Stevens LA, Schmid CH, Zhang YL, Castro AF 3rd, Feldman HI, et al.; CKD-EPI (Chronic Kidney Disease Epidemiology Collaboration). A new equation to estimate glomerular filtration rate. Ann Intern Med. 2009 May;150(9):604-12.

14 Thielmann M, Kottenberg E, Kleinbongard P, Wendt D, Gedik N, Pasa S, et al. Cardioprotective and prognostic effects of remote ischaemic preconditioning in patients undergoing coronary artery bypass surgery: a singlecentre randomised, double-blind, controlled trial. Lancet. 2013 Aug;382(9892):597-604.

15 Kellum JA, Sileanu FE, Bihorac A, Hoste EA, Chawla LS. Recovery after Acute Kidney Injury. Am J Respir Crit Care Med. 2017 Mar;195(6):784-91.

16 Palevsky PM, Molitoris BA, Okusa MD, Levin A, Waikar SS, Wald R, et al. Design of clinical trials in acute kidney injury: report from an NIDDK workshop on trial methodology. Clin J Am Soc Nephrol. 2012 May;7(5):844-50.

17 Hu J, Chen R, Liu S, Yu X, Zou J, Ding X. Global Incidence and Outcomes of Adult Patients With Acute Kidney Injury After Cardiac Surgery: A Systematic Review and Meta-Analysis. J Cardiothorac Vasc Anesth. 2016 Jan; 30(1):82-9.

18 Moore E, Tobin A, Reid D, Santamaria J, Paul E, Bellomo R. The Impact of Fluid Balance on the Detection, Classification and Outcome of Acute Kidney Injury After Cardiac Surgery. J Cardiothorac Vasc Anesth. 2015 Oct; 29(5):1229-35.

19 Jin J, Chang SC, Shen B, Xu J, Jiang W, Wang Y, et al. Usefulness of High Estimated Pulmonary Artery Systolic Pressure to Predict Acute Kidney Injury After Cardiac Valve Operations. Am J Cardiol. 2019 Feb;123(3): 440-5.

20 Janda S, Shahidi N, Gin K, Swiston J. Diagnostic accuracy of echocardiography for pulmonary hypertension: a systematic review and meta-analysis. Heart. 2011 Apr;97(8):612-22.

21 Beaubien-Souligny W, Benkreira A, Robillard P, Bouabdallaoui N, Chassé M, Desjardins G, et al. Alterations in Portal Vein Flow and Intrarenal Venous Flow Are Associated With Acute Kidney Injury After Cardiac Surgery: A Prospective Observational Cohort Study. J Am Heart Assoc. 2018 Oct;7(19):e009961.

22 Valdez GD, Mihos CG, Santana O, Heimowitz TB, Goldszer R, Lamas GA, et al. Incidence of postoperative acute kidney injury in patients with chronic kidney disease undergoing minimally invasive valve surgery. J Thorac Cardiovasc Surg. 2013 Dec;146(6):1488-93.

23 Biancari F, Rimpiläinen R. Meta-analysis of randomised trials comparing the effectiveness of miniaturised versus conventional cardiopulmonary bypass in adult cardiac surgery. Heart. 2009 Jun;95(12): 964-9.

24 Neumann FJ, Sousa-Uva M, Ahlsson A, Alfonso F, Banning AP, Benedetto U, et al.; ESC Scientific Document Group. 2018 ESC/EACTS Guidelines on myocardial revascularization. Eur Heart J. 2019 Jan; 40(2):87-165. 
25 Murzi M, Kallushi E, Tiwari KK, Cerillo AG, Bevilacqua S, Karimov JH, et al. Minimally invasive mitral valve surgery through right thoracotomy in patients with patent coronary artery bypass grafts. Interact Cardiovasc Thorac Surg. 2009 Jul;9(1):29-32.

26 Suri RM, Schaff HV, Meyer SR, Hargrove WC 3rd. Thoracoscopic versus open mitral valve repair: a propensity score analysis of early outcomes. Ann Thorac Surg. 2009 0ct;88(4):1185-90.

27 Khan UA, Coca SG, Hong K, Koyner JL, Garg AX, Passik CS, et al. Blood transfusions are associated with urinary biomarkers of kidney injury in cardiac surgery. J Thorac Cardiovasc Surg. 2014 Aug;148(2):726-32.

28 Oprea AD, Del Rio JM, Cooter M, Green CL, Karhausen JA, Nailer P, et al. Pre- and postoperative anemia, acute kidney injury, and mortality after coronary artery bypass grafting surgery: a retrospective observational study. Can J Anaesth. 2018 Jan;65(1):46-59.

29 Palomba H, de Castro I, Neto AL, Lage S, Yu L. Acute kidney injury prediction following elective cardiac surgery: AKICS Score. Kidney Int. 2007 Sep;72(5):624-31.

30 Jorge-Monjas P, Bustamante-Munguira J, Lorenzo M, Heredia-Rodríguez M, Fierro I, Gómez-Sánchez E, et al. Predicting cardiac surgery-associated acute kidney injury: the CRATE score. J Crit Care. 2016 Feb;31(1): $130-8$. 